\title{
Melatonin ameliorates ANIT-induced cholestasis by activating Nrf2 through a PI3K/Akt-dependent pathway in rats
}

\author{
YUNZHOU LI ${ }^{1 *}$, HAN YU $^{1 *}$, ZONGYING XU ${ }^{1 *}$, SHAOHUA SHI $^{1}$, DINGNAN WANG ${ }^{1}$, XINGHUA SHI $^{2}$, \\ YUCHEN WANG ${ }^{1}$, BAIHUI ZENG ${ }^{1}$, HUIFANG DENG ${ }^{1}$, XIULAN DENG ${ }^{2}$ and XIANGGEN ZHONG ${ }^{1}$ \\ ${ }^{1}$ Synopsis of Golden Chamber Department, ${ }^{2}$ Department of Pharmacology, \\ Chinese Medicine College, Beijing University of Chinese Medicine, Chaoyang, Beijing 100029, P.R. China
}

Received July 31, 2018; Accepted November 13, 2018

DOI: $10.3892 / \mathrm{mmr} .2018 .9746$

\begin{abstract}
Cholestasis is a devastating liver condition which is increasing in prevalence worldwide; however, its underlying pathogenic mechanisms remain to be fully elucidated. It was hypothesised that melatonin may alleviate the hepatic injury associated with cholestasis due to its established antioxidant effects. Therefore, the effect and potential anticholestatic properties of melatonin were investigated in rats with $\alpha$-naphthylisothiocyanate (ANIT)-induced liver injury, a common animal model that mimics the cholestasis-associated liverinjury in humans. The rats received intraperitonealinjection of ANIT with or without subsequent treatment with melatonin, and were sacrificed $24 \mathrm{~h}$ later. The serum biochemistry parameters of the liver were measured using conventional laboratory assays, and the liver tissue was subjected to conventional histological examination, reverse transcription-quantitative
\end{abstract}

Correspondence to: Professor Xianggen Zhong, Synopsis of Golden Chamber Department, Chinese Medicine College, Beijing University of Chinese Medicine, 11 North 3rd Ring East Road, Chaoyang, Beijing 100029, P.R. China

E-mail: zhongxg@bucm.edu.cn

Professor Xiulan Deng, Department of Pharmacology, Chinese Medicine College, Beijing University of Chinese Medicine, 11 North 3rd Ring East Road, Chaoyang, Beijing 100029, P.R. China

E-mail: dengxiulan@bucm.edu.cn

*Contributed equally

Abbreviations: ALP, alkaline phosphatase; ALT, alanine transaminase; ANIT, $\alpha$-naphthylisothiocyanate; AST, aspartate transaminase; b.w., body weight; CMC, carboxymethyl cellulose; DBIL, direct bilirubin; GCL, glutamate cysteine ligase; GGT, $\gamma$-glutamyl transferase; GS, glutathione synthase; GSH, glutathione; i.p., intraperitoneal; Nrf2, nuclear factor-erythroid 2-related factor-2; OS, oxidative stress; ROS, reactive oxygen species; TBA, total bile acid; TBIL, total bilirubin

Key words: melatonin, cholestasis, nuclear factor-erythroid 2-related factor-2, phosphoinositide-3 kinase/Akt-dependent pathway polymerase chain reaction analysis and western blotting. The levels of alanine transaminase, aspartate transaminase, total bilirubin, direct bilirubin, total bile acids, alkaline phosphatase, $\gamma$-glutamyl transferase and glutathione were restored in rats treated with melatonin. Histological examination provided further evidence supporting the protective effect of melatonin against ANIT-induced cholestasis. In addition, the mRNA and protein expression levels of glutamate cysteine ligase, phosphorylated Akt and nuclear factor-erythroid 2-related factor- 2 were restored in rats treated with melatonin. These findings indicate that melatonin is a natural agent that appears to be promising for the treatment of cholestasis, and that the anticholestatic effects of melatonin involve the alleviation of oxidative stress.

\section{Introduction}

Cholestasis is characterised by a reduction in bile flow and bile acid accumulation (1), and has a higher incidence in hepatopathy. The prevalence of cholestasis has been increasing globally in recent years, becoming a major public health concern. A previous study in Shanghai revealed that the total incidence of cholestasis was $10.26 \%$ among patients with chronic liver disease (2). A cross-sectional study of cholestasis in 1,000 patients with chronic viral hepatitis in China demonstrated that, following discharge of $56 \%$ of patients with chronic viral hepatitis from hospital, the main indicators of intrahepatic cholestasis, alkaline phosphatase (ALP) or $\gamma$-glutamyl transferase (GGT), were higher than the upper limit of normal, and the risk and severity of liver fibrosis and cirrhosis in these patients were markedly increased (3). There are several causes of cholestasis, which may be broadly classified into hepatocellular and obstructive. Obstructive cholestasis typically involves bile plugging of the interlobular bile ducts, portal expansion and bile duct proliferation in association with centrilobular cholate injury (3). In hepatocellular cholestasis, there is impaired hepatocellular bile secretion, resulting in intrahepatic accumulation of toxic bile components, including bile acids and bilirubin, leading to progressive liver injury $(4,5)$. During cholestasis, the role of oxidative stress in hepatocellular injury has become a focus of research interest (6); however, the precise pathogenic mechanisms remain to be fully elucidated. 
$\alpha$-naphthylisothiocyanate (ANIT) is a hepatotoxin known to cause intrahepatic cholestasis due to selective damage of the bile duct epithelial cells. These cells, in turn, release factors that attract neutrophils, which then injure hepatocytes $(7,8)$, leading to intrahepatic cholestasis that is pathologically similar to drug-induced cholangiolitic hepatitis in humans (9). Therefore, the administration of ANIT to experimental animals, including rats, mice and guinea pigs, may be used to generate a model accurately mimicking intrahepatic cholestasis and hepatic damage in humans $(10,11)$.

Melatonin is a methoxyindole that is principally synthesised and secreted by the pineal gland at night under normal light/dark cycles $(12,13)$. Melatonin is also key in a number of physiological and cellular processes, including immune response, antioxidant defence, haemostasis and glucose regulation, depending on the melatonin signalling pathway involved $(14,15)$. In addition to its functions as a hormone, melatonin exerts antioxidant effects by scavenging reactive oxygen species (ROS) and by inhibiting lipid peroxidation (16-18), and has also been reported to possess anti-inflammatory properties (19). Melatonin has also emerged as a valuable biomarker for estimating the serotonin status in the brain, particularly for treatment monitoring purposes (20). Evidence indicates that the prolonged administration of melatonin attenuates the increase in total and low-density lipoprotein cholesterol concentration and the decrease in high-density lipoprotein cholesterol concentration in the serum of rats fed a hypercholesterolemic diet (21). In addition, orally administered melatonin was found to reduce the increase in serum total cholesterol concentration and attenuate the disruption of serum cholesterol status in rats with ANIT-induced acute liver injury with cholestasis; this protective effect may be due to its antioxidant action and its inhibitory action against neutrophil infiltration (22-24). However, the effects and potential mechanism of action of melatonin in the context of cholestasis remain to be fully elucidated.

The antioxidant defence system includes non-enzymatic and enzymatic components (25), with the latter dominated by superoxide dismutase, catalase and glutathione peroxidase (26). These antioxidant defence-related enzymes are modulated by nuclear factor-erythroid 2-related factor-2 (Nrf2) (27), which is central to the protection of cells against oxidative and/or xenobiotic damage by binding to genomic antioxidant response elements and stimulating the expression of phase II antioxidant genes $(28,29)$. In another study, Paeonia lactiflora pall and paeoniflorin alleviated ANIT-induced cholestasis by activating Nrf2 through the phosphoinositide-3 kinase (PI3K)/Akt-dependent pathway $(30,31)$.

The role of melatonin in cholestasis may be associated with resistance to oxidative stress. Although the mechanism underlying the action of melatonin in the treatment of liver disease has been widely investigated, the mechanisms underlying the alleviation of oxidative stress through the PI3K/Akt signalling pathway and Nrf2 remain to be fully elucidated. The aim of the present study was to investigate whether melatonin can alleviate ANIT-induced cholestasis. Furthermore, through metabonomics investigation of the function of melatonin against ANIT-induced cholestasis, glutathione (GSH) synthetic enzymes may be the one of the potential biomarkers. The changes in the expression of GSH synthetic enzymes were investigated in rats with ANIT-induced cholestasis under treatment with melatonin, as were the possible contributions of the PI3K/Akt signalling pathway and Nrf2. These findings may provide novel insight into the mechanisms underlying the development of cholestasis and indicate a novel therapeutic approach to this condition.

\section{Materials and methods}

Animals and treatments. A total of 18 male Sprague-Dawley rats (7-8 weeks old; weighing $260 \pm 20 \mathrm{~g}$ ) were obtained from SPF (Beijing) Biotechnology Co., Ltd. (Beijing, China; certification no. SCXK-JING 2016-0002). All animals were allowed to acclimate for 1 week prior to the experiments and were maintained at a constant temperature $\left(25 \pm 2^{\circ} \mathrm{C}\right)$ and $50 \%$ humidity with a 12:12-h light/dark cycle; all the rats had access to water and food ad libitum. The study protocol was performed in strict accordance with the recommendations of the Guidelines for the Care and Use of Laboratory Animals of the Ministry of Science and Technology of China, and was approved by Beijing University of Chinese Medicine Medical and Experimental Animal Ethics Committee (Beijing, China) with certification no. bucm-4-2017122735-4035.

An overview of the experimental design is presented in Table I. In brief, the rats were randomly divided into three groups ( $n=6$ per group) as follows: Control group, in which the rats were treated with vehicle $[75 \mathrm{mg} / \mathrm{kg}$ body weight (b.w.) olive oil] alone; ANIT group, in which the rats received intraperitoneal (i.p.) injection of ANIT (Sigma; Merck KGaA, Darmstadt, Germany) at a dose of $75 \mathrm{mg} / \mathrm{kg}$ b.w.; and the melatonin + ANIT group, in which the rats received melatonin $(100 \mathrm{mg} / \mathrm{kg}$ b.w.; Sigma; Merck KGaA) orally $12 \mathrm{~h}$ following the initial ANIT injection. Instead of melatonin, rats in the control and ANIT groups were orally administered with the same volume of $0.25 \%$ carboxymethyl cellulose (CMC) sodium (Yuanye Biological Technology Co., Ltd., Shanghai, China) $12 \mathrm{~h}$ after the initial injection. All rats were fasted for $12 \mathrm{~h}$ prior to the injections, and each rat was weighed prior to the i.p. injection of ANIT and oral administration of melatonin or CMC sodium. ANIT was dissolved in olive oil at a dose of $75 \mathrm{mg} / \mathrm{kg}$ b.w., i.e., $1 \mathrm{ml}$ of ANIT solution in olive oil $(75 \mathrm{mg} / \mathrm{ml})$ per $100 \mathrm{~g}$ b.w., to induce liver injury associated with cholestasis, as previously described (23). Melatonin $(100 \mathrm{mg} / \mathrm{kg}$ b.w.) was suspended in $1 \mathrm{ml}$ of $0.25 \% \mathrm{CMC}$ sodium.

Sample collection and liver function assays. The rats were provided with standard chow and water following treatment completion. According to pilot experiments, the rats were then fasted for $12 \mathrm{~h}$, and were sacrificed $36 \mathrm{~h}$ after the initial ANIT or vehicle injection. Blood samples were collected from the inferior vena cava and the livers were immediately removed. All efforts were made to minimise animal suffering. The serum ALP (cat. no. A059-1), aspartate aminotransferase (AST; cat. no. C010-2), alanine aminotransferase (ALT; cat. no. C0009-2), GGT (cat. no. C017-1), total bilirubin (TBIL; cat. no. C019-1), direct bilirubin (DBIL; cat. no. C019-2) and total bile acid (TBA; cat. no. E003-1) levels were detected using chemical oxidation assays. All assay kits were purchased from Nanjing Jiancheng Bioengineering Institute (Nanjing, China). 
Table I. Stages of the animal experimental design.

\begin{tabular}{lccccr}
\hline & \multicolumn{5}{c}{ Procedure/treatment } \\
\cline { 2 - 6 } Group & $0 \mathrm{~h}$ & $12 \mathrm{~h}$ & $24 \mathrm{~h}$ & $36 \mathrm{~h}$ & $48 \mathrm{~h}$ \\
\hline Control & Fast & Olive oil & $0.25 \% \mathrm{CMC}$ & Fast & Sacrifice \\
Model & Fast & $75 \mathrm{mg} / \mathrm{kg} \mathrm{ANIT}$ & $0.25 \% \mathrm{CMC}$ & Fast & Sacrifice \\
Melatonin & Fast & $75 \mathrm{mg} / \mathrm{kg}$ ANIT & $100 \mathrm{mg} / \mathrm{kg}$ melatonin & Fast & Sacrifice
\end{tabular}

ANIT, $\alpha$-naphthylisothiocyanate.

Histological assessment of liver damage. The liver tissues were excised and fixed in 10\% phosphate-buffered formalin. The fixed issues were cut into $1 \times 1 \times 0.3-\mathrm{cm}$ sections, dehydrated in a graded series of alcohol and embedded in paraffin blocks. The blocks were then cut into 4-5- $\mu \mathrm{m}$ sections, dewaxed in xylene, dipped in haematoxylin and agitated for $30 \mathrm{sec}$, rinsed in $\mathrm{H}_{2} \mathrm{O}$ for $1 \mathrm{~min}$, followed by staining with $1 \%$ eosin $\mathrm{Y}$ solution for $30 \mathrm{sec}$ with agitation, all at room temperature $\left(20-25^{\circ} \mathrm{C}\right)$. The slides were then examined under a BX53 microscope (Olympus Corporation, Tokyo, Japan).

GSH assay in the liver. The active GSH was determined with a commercial kit (cat. no. A006-1; Nanjing Jiancheng Bioengineering Institute) according to the manufacturer's protocol. In brief, a portion of the liver tissue was homogenised by adding nine volumes of saline. The homogenates were centrifuged at $3,000 \mathrm{x} \mathrm{g}$ and $4^{\circ} \mathrm{C}$ for $10 \mathrm{~min}$ to collect the supernatant. The reagents were added to $0.5 \mathrm{ml}$ supernatant according to the instructions, centrifuged $3,500 \times \mathrm{g}\left(4^{\circ} \mathrm{C}\right)$ for $10 \mathrm{~min}$, and $1 \mathrm{ml}$ supernatant was collected for the chromogenic reaction.

Western blot analysis. Nuclear and cytoplasmic extractions were accomplished using the Nuclear and Cytoplasmic Extraction kit (Biosynthesis Biotechnology Company, Beijing, China) according to the manufacturer's protocol, and then assayed for protein levels of glutamate cysteine ligase (GCL), Akt, and Nrf2 with western blotting using an automated capillary-based size sorting system (Automated Capillary Western Blot, ProteinSimple, San Jose, CA, USA). All procedures were performed with the reagents included in the kit and according to the manufacturer's protocol. In brief, $8 \mu \mathrm{l}$ of diluted protein lysate was mixed with $2 \mu \mathrm{l}$ of $5 \mathrm{X}$ fluorescent Master mix and heated at $95^{\circ} \mathrm{C}$ for $5 \mathrm{~min}$. The samples, blocking reagent, wash buffer, primary antibodies, secondary antibodies, and chemiluminescent substrate were dispensed into designated wells in a microplate provided by the manufacturer. The plate was loaded into the instrument and protein was drawn into individual capillaries on a 25-capillary cassette provided by the manufacturer. Protein separation with the resulting chemiluminescent signal was performed automatically on the individual capillaries using default settings. The data were analysed using Compass software (version 3.1.7; ProteinSimple, San Jose, CA, USA). The GCL catalytic subunit (GCLC), GLC modifier subunit (GCLM) and Nrf2 antibodies used were obtained from Abcam (1:50; cat. nos. ab80841, ab124827 and ab31163, respectively; Abcam, Cambridge, UK); Akt and $\beta$-actin were obtained from Cell signalling Technology (1:50; cat. nos. 4691 and 4970, respectively; CST, Danvers, MA, USA) and used as a loading control. Secondary antibodies used were obtained from ProteinSimple (1:1; cat. no. 042-206). Primary and secondary antibodies were incubated at room temperature for $30 \mathrm{~min}$.

RNA extraction and reverse transcription-quantitative polymerase chain reaction ( $R T-q P C R)$ analysis. Total RNA was extracted from the liver tissues using the mirVana miRNA Isolation kit (cat. no. AM1561, Ambion; Thermo Fisher Scientific, Inc., Waltham, MA, USA) according to the manufacturer's protocol. The yield of RNA was determined using a NanoDrop 2000 spectrophotometer (Thermo Fisher Scientific, Inc.) and the integrity was evaluated using agarose gel electrophoresis stained with ethidium bromide.

Quantification was performed with a two-step reaction process: Reverse transcription and PCR. Each reverse transcription reaction involved two steps. In the first step, $0.5 \mu \mathrm{g}$ RNA and $2 \mu \mathrm{l} 4 \mathrm{X}$ gDNA wiper mix were combined, and nuclease-free $\mathrm{H}_{2} \mathrm{O}$ was added up to $8 \mu \mathrm{l}$. The reactions were performed in a GeneAmp ${ }^{\circledR}$ PCR System 9700 (Applied Biosystems; Thermo Fisher Scientific, Inc.) for $2 \mathrm{~min}$ at $42^{\circ} \mathrm{C}$. In the second step, $2 \mu \mathrm{l}$ of $5 \mathrm{X}$ HiScript II Q RT SuperMix IIa was added to the mixture, and the reactions were run for $10 \mathrm{~min}$ at $25^{\circ} \mathrm{C}, 30 \mathrm{~min}$ at $50^{\circ} \mathrm{C}$, and $5 \mathrm{~min}$ at $85^{\circ} \mathrm{C}$. The $10-\mu l$ reverse transcription reaction mix was then diluted 10 times in nuclease-free $\mathrm{H}_{2} \mathrm{O}$ and maintained at $-20^{\circ} \mathrm{C}$. qPCR was performed using a LightCycler ${ }^{\circledR}$ 480 II Real-time PCR instrument (Roche Diagnostics, Basel, Switzerland) with a $10-\mu 1$ PCR mixture that included $1 \mu 1$ cDNA, $5 \mu$ l 2X QuantiFast ${ }^{\circledR}$ SYBR $^{\circledR}$ Green PCR Master mix (Qiagen GmbH, Hilden, Germany), $0.2 \mu \mathrm{l}$ forward primer, $0.2 \mu \mathrm{l}$ reverse primer and $3.6 \mu 1$ nuclease-free water. The reactions were incubated in a 384-well optical plate (Roche Diagnostics) at $95^{\circ} \mathrm{C}$ for $5 \mathrm{~min}$, followed by 40 cycles at $95^{\circ} \mathrm{C}$ for $10 \mathrm{sec}$ and at $60^{\circ} \mathrm{C}$ for $30 \mathrm{sec}$. Each sample was run in triplicate. At the end of the PCR cycles, melting curve analysis was performed to validate the specific generation of the expected PCR product. The primer sequences (Table II) were designed in the laboratory and synthesised by Generay Biotech (Shanghai, China) based on mRNA sequences obtained from the National Center for Biotechnology Information database.

The expression levels of the target mRNAs were normalised to those of $\beta$-actin and calculated using the $2^{-\Delta \Delta C q}$ method (32).

Statistical analysis. All statistical analyses were conducted using SPSS 20.0 software (IBM Corp., Armonk, NY, USA). 
Table II. Primer sequences for reverse transcription-quantitative polymerase chain reaction.

\begin{tabular}{lll}
\hline Gene & \multicolumn{1}{c}{ Forward primer $\left(5^{\prime}-3^{\prime}\right)$} & Reverse primer $\left(5^{\prime}-3^{\prime}\right)$ \\
\hline GCLC & CCAGGGTGATCCTCTCATAC & TGCCACTTTCATGTTCTCG \\
GCLM & CCACCAGATTTGACTGCAT & TTGCCTCAGAGAGCAGTTC \\
pAkt & AGAACCTCATGCTGGACA & CCTTGATACCCTCCTTGC \\
Nrf2 & TGGGTTCAGTGACTCGGA & TGTTGGCTGTGCTTTAGG \\
-actin & CCACCATGTACCCAGGCATT & CGGACTCATCGTACTCCTGC
\end{tabular}

GCLC, glutamate cysteine ligase catalytic subunit; GCLM, glutamate cysteine ligase modifier subunit; pAkt, phosphorylated Akt; Nrf2, nuclear factor-erythroid 2-related factor-2.
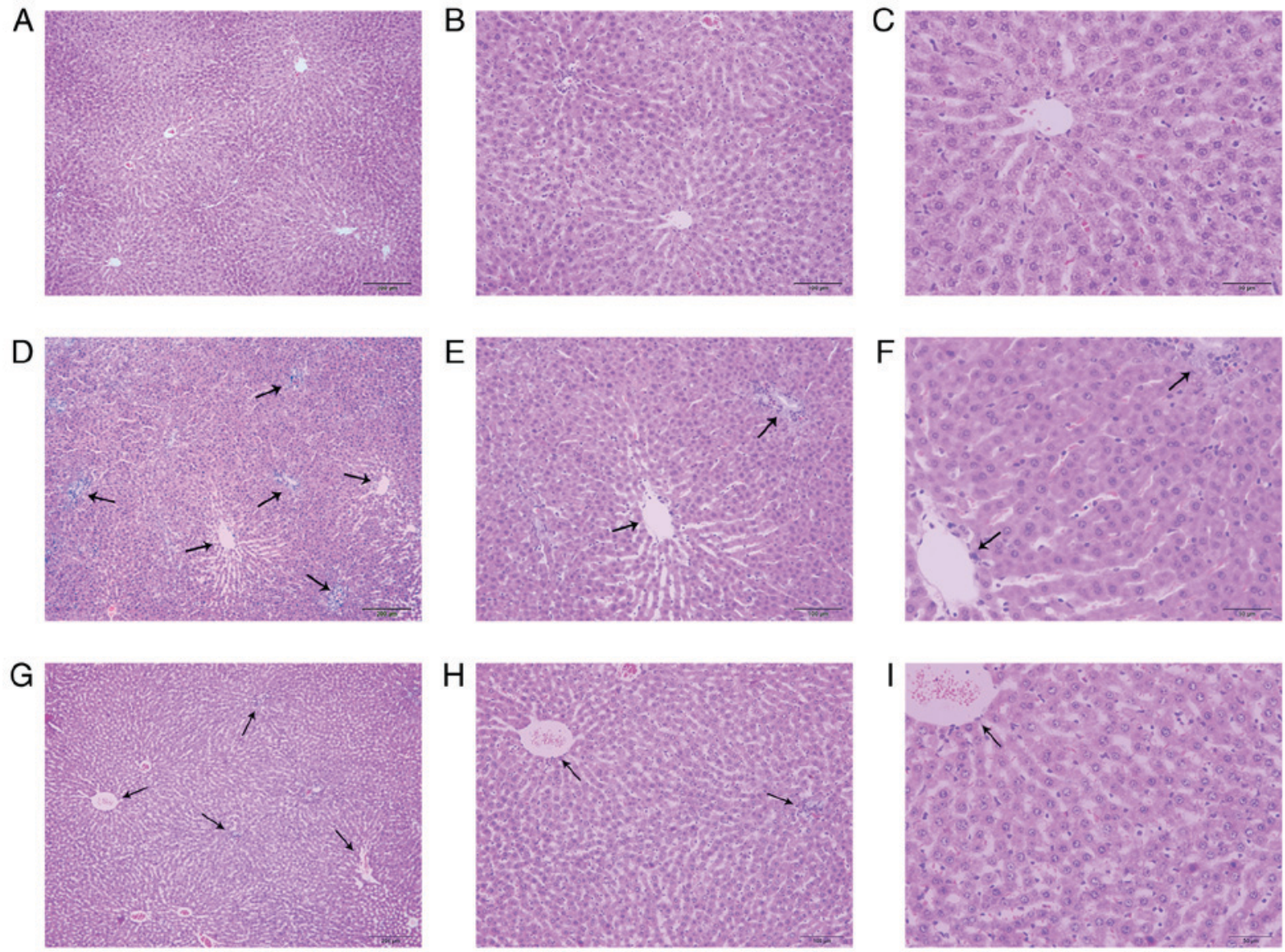

Figure 1. Melatonin reverses histological damage in the liver tissues of rats with $\alpha$-naphthyl isothiocyanate-induced cholestasis. Control group images at magnifications of (A) x50, (B) x100 and (C) x200; cholestasis model group images at magnifications of (D) x50, (E) x100 and (F) x200; and melatonin treatment group images at magnifications of $(\mathrm{G}) \times 50,(\mathrm{H})$ x100 and (I) x200. Liver tissue histological damage is indicated by black arrows.

All experiments were repeated at least three times and the obtained data are presented as the mean \pm standard deviation. Student's t-test was used for the analysis of statistical significance between two groups, and one-way analysis of variance followed by Dunnett's post hoc test was applied to analyse statistical significance among three groups or more. $\mathrm{P}<0.05$ was considered to indicate a statistically significant difference.

\section{Results}

Histological examination. Representative photomicrographs of the haematoxylin and eosin-stained liver tissues from the control,
ANIT and ANIT + melatonin groups are shown in Fig. 1. The control group exhibited a normal lobular architecture with central veins and radiating hepatic cords (Fig. 1A-C), whereas the ANIT group exhibited severe changes in liver morphology, including acute infiltration by neutrophils, metamorphosis, sinusoid congestion and hepatic necrosis and inflammation (Fig. 1D-F). However, the model rats treated with melatonin exhibited only mild bile duct epithelial damage and hepatocyte hydropic degeneration, and relatively milder neutrophil infiltration (Fig. 1G-I).

Effects of melatonin on serum biochemistry. As shown in Fig. 2, the ANIT-treated rats exhibited a marked increase 

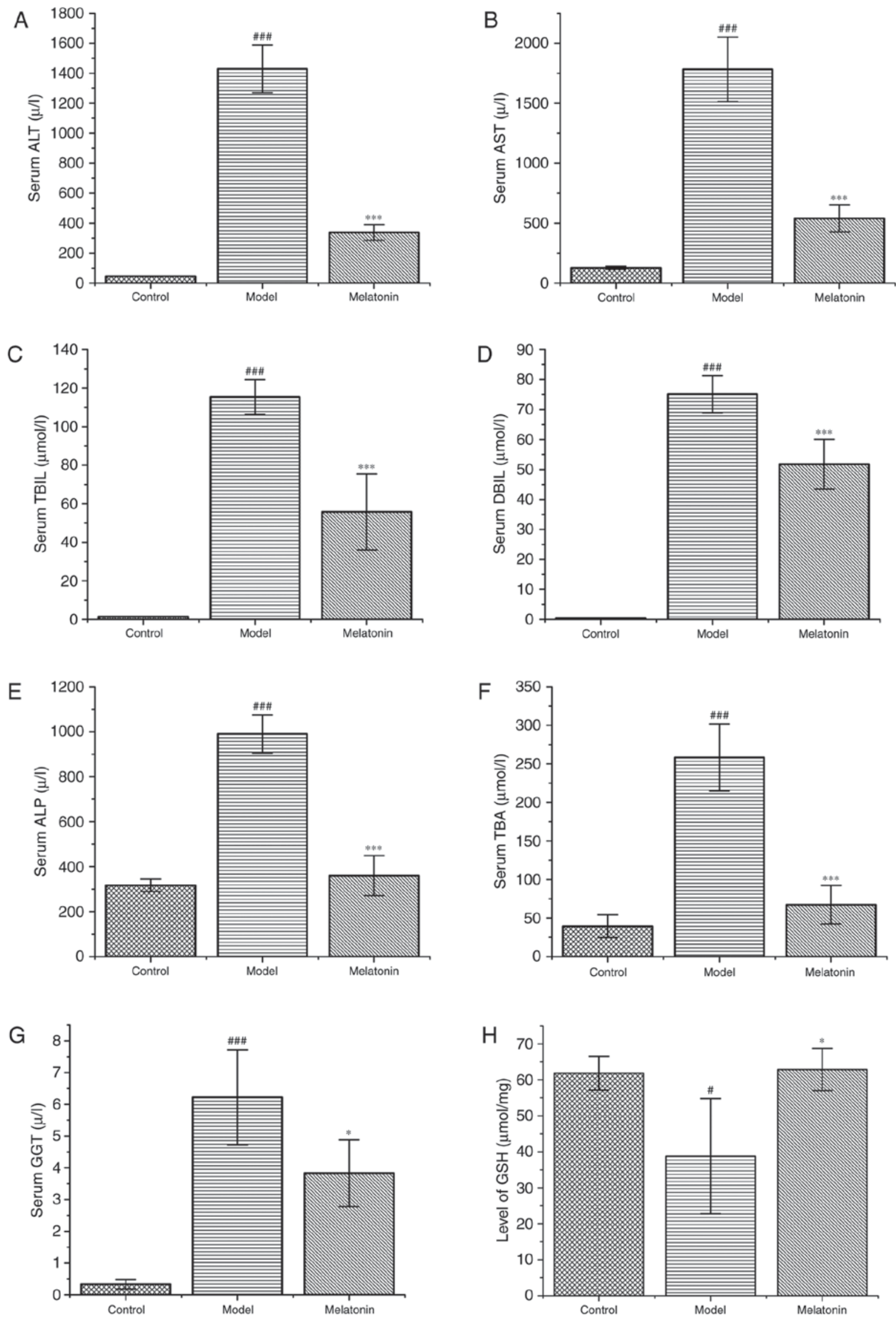

Figure 2. Effects of melatonin on serum biochemistry. The rats were treated with ANIT (75 mg/kg) with and without melatonin. The following liver function markers in the serum were assayed: (A) ALT, (B) AST, (C) TBIL, (D) DBIL, (E) ALP, (F) TBA, and (G) GGT. (H) Levels of GSH in liver tissues. Data are expressed as the mean \pm standard error of the mean $\left(\mathrm{n}=6\right.$ per group). ${ }^{\# \#} \mathrm{P}<0.001$ and ${ }^{\#} \mathrm{P}<0.05$ compared with the control group; ${ }^{* * *} \mathrm{P}<0.001$ and ${ }^{*} \mathrm{P}<0.05$ compared with the ANIT group. ANIT, $\alpha$-naphthyl isothiocyanate; ALT, alanine transaminase; AST, aspartate transaminase; TBIL, total bilirubin; DBIL, direct bilirubin; ALP, alkaline phosphatase; TBA, total bile acids; GGT, $\gamma$-glutamyl transferase; GSH, glutathione. 
A

A $\quad \beta$-actin

kes CON MOD MT

GCLC

CON MOD MT

GCLM

pAkt

CON MOD MT

CON MOD MT

Nrf2
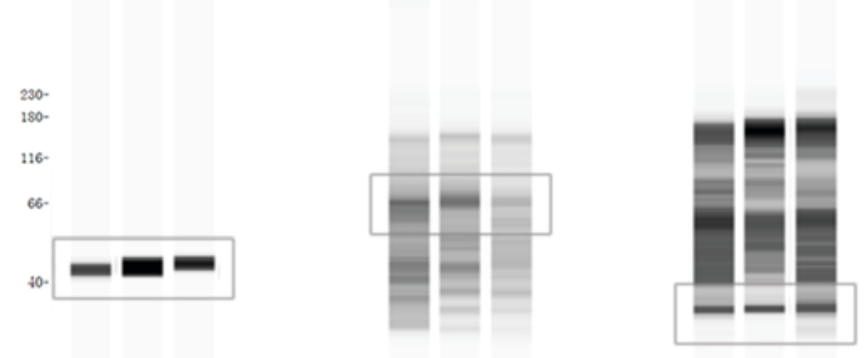

CON MOD MT

B

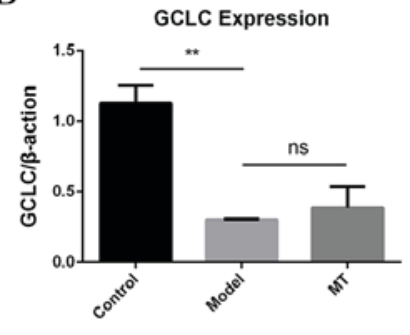

F

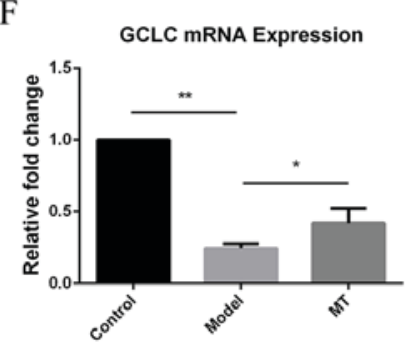

C

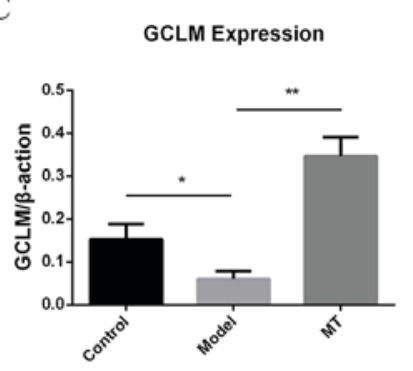

G

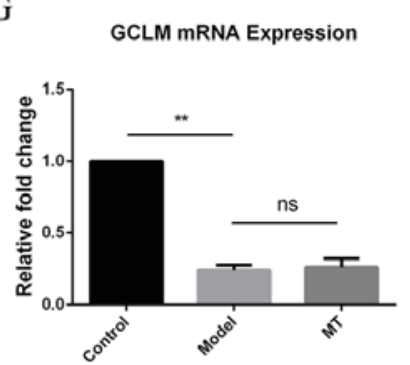

D

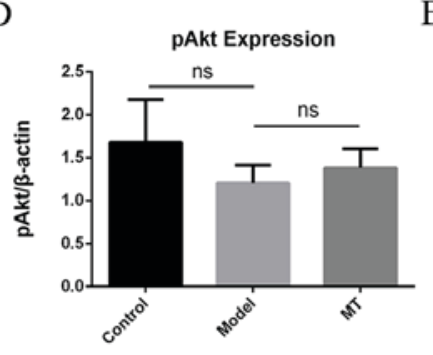

$\mathrm{H}$

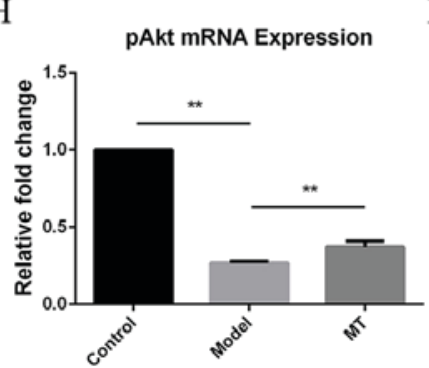

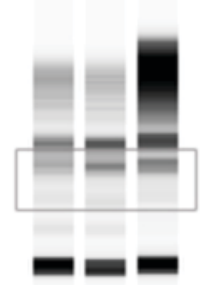

E

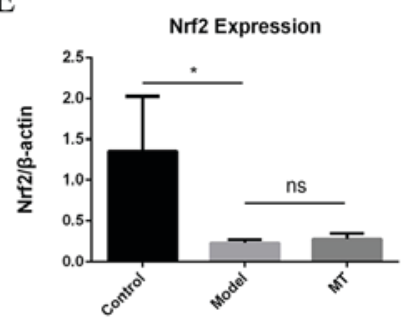

I

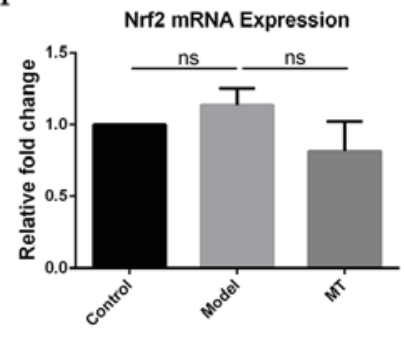

Figure 3. Effects of melatonin on hepatic protein and mRNA expression. (A) Effect of melatonin on hepatic protein expression. Effect of melatonin on hepatic protein expression of (B) GCLC, (C) GCLM, (D) pAkt and (E) Nrf2. Effect of melatonin on hepatic mRNA expression of (F) GCLC, (G) GCLM, (H) pAkt and (I) Nrf2. ${ }^{* *} \mathrm{P}<0.01$ and ${ }^{*} \mathrm{P}<0.05$. CON, control; MOD, model; MT, melatonin; GCLC, glutamate cysteine ligase catalytic subunit; GCLM, glutamate cysteine ligase modifier subunit; Nrf2, nuclear factor-erythroid 2-related factor-2; ns, not significant.

in ALT and AST levels, which were significantly reduced following treatment with melatonin (Fig. 2A and B). Similarly, the levels of TBIL, DBIL, ALP, TBA and GGT were markedly increased in ANIT-treated rats compared with the control group, and were effectively reduced following melatonin administration (Fig. 2C-G).

Effects of melatonin on hepatic GSH. Compared with the control group, the model groups exhibited markedly reduced concentrations of GSH in the liver tissue; the GSH levels were increased in the melatonin-treated group compared with those in the ANIT group (Fig. 2H).
Effects of melatonin on the activation of GCLC, Nrf2, Akt and GCLM. The results of protein analysis (Fig. 3A-E) showed that melatonin upregulated the protein expression of GCLM. Melatonin increased the mRNA levels of GCLC and pAkt, the expression levels of which were reduced by administration of ANIT (Fig. 3F-I).

\section{Discussion}

Serum liver enzymes (including ALT, AST, ALP and GGT), TBA and serum bilirubin (TBIL and DBIL) are important indices of the clinical manifestations of cholestatic hepatitis. 


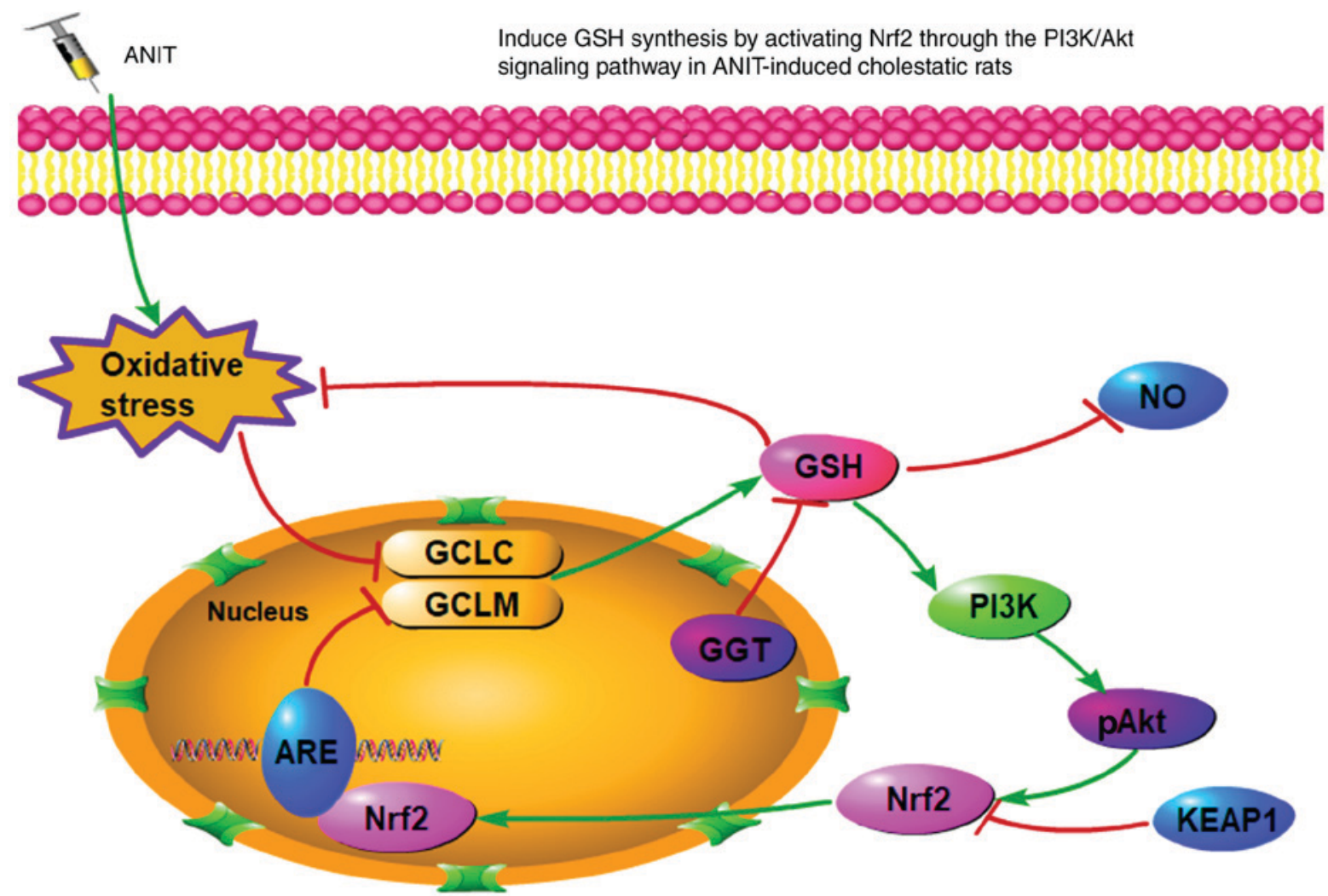

Figure 4. Schematic presentation of the proposed effects of melatonin against ANIT-induced intrahepatic cholestasis. ANIT, $\alpha$-naphthyl isothiocyanate; GCLC, glutamate cysteine ligase catalytic subunit; GCLM, glutamate cysteine ligase modifier subunit; Nrf2, nuclear factor-erythroid 2-related factor-2; ARE, antioxidant response element; GSH, glutathione; GGT, $\gamma$-glutamyl transferase; PI3K, phosphoinositide-3 kinase; KEAP1, Kelch-like ECH-associated protein 1.

These markers tend to increase following ANIT administration and commonly peak at $48 \mathrm{~h}(33)$. The results of the present study indicate that treatment with melatonin notably decreased the ANIT-induced serum levels of serum ALT, AST, TBA, TBIL, DBIL and ALP. Furthermore, the histological manifestations of liver damage were reduced following melatonin treatment. Overall, these results indicate that melatonin may be a candidate drug exerting protective effects against ANIT-induced cholestasis and ensuing liver injury.

The activation of Nrf2 protects the liver from xenobiotic toxicity by regulating the expression of several detoxifying and antioxidant enzymes, in addition to transporters (34-37). The PI3K/Akt pathway has been suggested as the key signalling pathway in this system by regulating the expression of $\mathrm{Nrf} 2$, with the activity of the GCL subunit in hepatocytes also regulated by PI3K/Akt signalling (38). GSH is involved in the detoxification of chemical substances conjugated by the catalytic action of GSH and the extracellular transport of conjugated compounds, acting as one of the major cellular antioxidant defence molecules against ROS production (39). GSH synthesis occurs in the cytosol of all mammalian cells via two enzymatic steps: The formation of $\gamma$-glutamylcysteine from glutamate and cysteine catalysed by GCL, and the formation of GSH from $\gamma$-glutamylcysteine and glycine catalysed by GSH synthase (40). Akt, as a major regulator of PI3K signalling, exerts an anti-apoptotic effect, and may be phosphorylated and activated during several different types of cell death (41). Nrf2 acts as a key transcription factor and a regulator of the expression of GCL in response to oxidative stress, along with other anti-oxidative stress genes. Therefore, the present study also examined the effects of melatonin on the expression of GSH and its synthetic enzymes. It was demonstrated that melatonin markedly increased the levels of GSH, and upregulated the mRNA and protein expression levels of GCLC and GCLM. These findings indicated that the effect of melatonin on the increase in GSH may be associated with upregulation of the expression of GCLM and GCLC, thereby contributing to the protection of cells against oxidative damage and against ANIT-induced cholestasis. A schematic summary of the proposed effects of melatonin against ANIT-induced intrahepatic cholestasis is presented in Fig. 4. Overall, the results of the present study indicate that melatonin markedly attenuated cholestasis through regulating oxidative stress via targeting the Nrf2-PIK/Akt axis, highlighting this natural product as an antioxidant candidate for the treatment of cholestasis.

\section{Acknowledgements}

The authors would like to thank the staff of the Science Center Department of Beijing University of Chinese Medicine (Beijing, China), the Pathology Department of China-Japan Friendship Hospital (Beijing, China), Shanghai OE Biotech, Inc. (Shanghai, China) and Professor Jian Li (Department of Pharmacology, Chinese Medicine College, Beijing University of Chinese Medicine, Beijing, China) and Mrs. Shujing Zhang (Science Center Department of Beijing University of Chinese Medicine, Beijing, China) for their technical support. 


\section{Funding}

The present study was supported by National Natural Science Foundation Project of China (grant no. 81573963).

\section{Availability of data and materials}

The datasets generated and analysed during the present study are available from the corresponding author on reasonable request.

\section{Authors' contributions}

XZ and XD conceived and designed the study. YL, HY and ZX acquired, analysed and interpreted the data. DW and SS were responsible for handling the animals and obtaining tissue specimens. XS and YW performed statistical analyses. BZ and HD performed histopathological analyses. All authors have read and approved the final version of this manuscript.

\section{Ethics approval and consent to participate}

The study protocol was in strict accordance with the recommendations of the Guidelines for the Care and Use of Laboratory Animals of the Ministry of Science and Technology of China, and was approved by Beijing University of Chinese Medicine Medical and Experimental Animal Ethics Committee (Beijing, China). All efforts were made to minimise animal suffering.

\section{Patient consent for publication}

Not applicable.

\section{Competing interests}

The authors declare that they have no competing interests.

\section{References}

1. Yang K, Köck K, Sedykh A, Tropsha A and Brouwe KL: An updated review on drug-induced cholestasis: Mechanisms and investigation of physicochemical properties and pharmacokinetic parameters. J Pharm Sci 102: 3037-3057, 2013.

2. Cao X, Gao Y, Zhang W, Xu P, Fu Q, Chen C, Li C, Yang C, $\mathrm{Ma} \mathrm{G}, \mathrm{Qu}$ Y, et al: Cholestasis morbidity rate in first-hospitalized patients with chronic liver disease in Shanghai. Zhonghua Gan Zang Bing Za Zhi 23: 569-573, 2015 (In Chinese).

3. Cheng J, Wang J, Zhang W, Yang X and Cao Y: A cross-sectional study on intrahepatic cholestasis indicators of viral hepatitis patients. J Hepatol 62 (Suppl 2): pp0592, 2015.

4. Park HW, Lee NM, Kim JH, Kim KS and Kim SN: Parenteral fish oil-containing lipid emulsions may reverse parenteral nutrition-associated cholestasis in neonates: A systematic review and meta-analysis. J Nutr 145: 277-283, 2015.

5. Trauner M, Meier PJ and Boyer JL: Molecular pathogenesis of cholestasis. N Engl J Med 339, 1217-1227, 1998.

6. Wagner M, Zollner G and Trauner M: New molecular insights into the mechanisms of cholestasis. J Hepatol 51: 565-580, 2009.

7. Copple BL, Jaeschke H and Klaassen CD: Oxidative stress and the pathogenesis of cholestasis. Semin Liver Dis 30: 195-204, 2010.

8. Hill DA and Roth RA: Alpha-naphthylisothiocyanate causes neutrophils to release factors that are cytotoxic to hepatocytes. Toxicol Appl Pharmacol 148: 169-175, 1998.
9. Hill DA, Jean PA and Roth RA: Bile duct epithelial cells exposed to alpha-naphthylisothiocyanate produces a factor that causes neutrophil-dependent hepatocellular injury in vitro. Toxicol Sci 47: 118-125, 1999.

10. Waters NJ, Holmes E, Williams A, Waterfield CJ, Farrant RD and Nicholson JK: NMR and pattern recognition studies on the time-related metabolic effects of alpha-naphthylisothiocyanate on liver, urine, and plasma in the rat: An integrative metabonomic approach. Chem Res Toxicol 14: 1401-1412, 2001.

11. Capizzo F and Roberts RJ: -Naphthylisothiocyanate (ANIT)-induced hepatotoxicity and disposition in various species. Toxicol Appl Pharmacol 19: 176-187, 1971.

12. Plaa GL and Priestly BG: Intrahepatic cholestasis induced by drugs and chemicals. Pharmacol Rev 28: 207-273, 1976.

13. Acuña-Castroviejo D, Escames G, Venegas C, Díaz-Casado ME, Lima-Cabello E, López LC, Rosales-Corral S, Tan DX and Reiter RJ: Extrapineal melatonin: Sources, regulation, and potential functions. Cell Mol Life Sci 71: 2997-3025, 2014.

14. Pääkkönen T, Mäkinen TM, Leppäluoto J, Vakkuri O, Rintamäki H, Palinkas LA and Hassi J: Urinary melatonin: A noninvasive method to follow human pineal function as studied in three experimental conditions. J Pineal Res 40: 110-115, 2006.

15. Claustrat B and Leston J: Melatonin: Physiological effects in humans. Neurochirurgie 61: 77-84, 2015.

16. Calvo JR, González-Yanes $\mathrm{C}$ and Maldonado MD: The role of melatonin in the cells of the innate immunity: A review. J Pineal Res 55: 103-120, 2013.

17. Zang LY, Cosma G, Gardner H and Vallyathan V: Scavenging of reactive oxygen species by melatonin. Biochim Biophys Acta 1425: 469-477, 1998.

18. Longoni B, Salgo MG, Pryor WA and Marchiafava PL: Effects of melatonin on lipid peroxidation by oxygen radicals. Life Sci 62: 853-859, 1998.

19. Allegra M, Reiter RJ, Tan DX, Gentile C, Tesoriere L and Livrea MA: The chemistry of melatonin's interaction with reactive species. J Pineal Res 34: 1-10, 2003.

20. Lotufo CM, Lopes C, Dubocovich ML, Farsky SH and Markus RP: Melatonin and $\mathrm{N}$-acetylserotonin inhibit leukocyte rolling and adhesion to rat microcirculation. Eur J Pharmacol 430: 351-357, 2001.

21. Batllori M, Molero-Luis M, Arrabal L, Heras JL, Fernandez-Ramos JA, Gutiérrez-Solana LG, Ibáñez-Micó S, Domingo R, Campistol J, Ormazabal A, et al: Urinary sulphatoxymelatonin as a biomarker of serotonin status in biogenic amine-deficient patients. Sci Rep 7: 14675, 2017.

22. Hoyos M, Guerreo JM, Perez-Cano R, Olivan J, Fabiani F, Garcia-Pergañeda A and Osuna C: Serum cholesterol and lipid peroxidation decreased by melatonin in diet-induced hypercholesterolemic rats. J Pineal Res 28: 150-155, 2000.

23. Ohta Y, Kongo M, Sasaki E, Ishiguro I and Harada N: Protective effect of melatonin against alpha-naphthylisothiocyanate-induced liver injury in rats. J Pineal Res 29: 15-23, 2000.

24. Jung KH, Hong SW, Zheng HM, Lee DH and Hong SS: Melatonin downregulates nuclear erythroid 2-related factor 2 and nuclear factor-kappaB during prevention of oxidative liver injury in a dimethylnitrosamine model. J Pineal Res 47: 173-183, 2009.

25. Ohta Y, Kongo-Nishimura M, Imai Y and Kitagawa A: Melatonin attenuates disruption of serum cholesterol status in rats with a single alpha-naphthylisothiocyanate treatment. J Pineal Res 42: 159-165, 2007.

26. Ding RB, Tian K, Cao YW, Bao JL, Wang M, He C, Hu Y, Su H and Wan JB: Protective effect of panax notoginseng saponins on acute ethanol-induced liver injury is associated with ameliorating hepatic lipid accumulation and reducing ethanol-mediated oxidative stress. J Agric Food Chem 63: 2413-2422, 2015.

27. Han D, Hanawa N, Saberi B and Kaplowitz N: Mechanisms of liver injury. III. Role of glutathione redox status in liver injury. Am J Physiol Gastrointest Liver Physiol 291: G1-G7, 2006.

28. Copple IM, Goldring CE, Kitteringham NR and Park BK: The Nrf2-Keap1 defence pathway: Role in protection against drug-induced toxicity. Toxicology 246: 24-33, 2008.

29. Kensler TW and Wakabayashi N: Nrf2: Friend or foe for chemoprevention? Carcinogenesis 31: 90-99, 2010.

30. Chen Z, Ma X, Zhu Y, Zhao Y, Wang J, Li R, Chen C, Wei S, Jiao W, Zhang Y, et al: Paeoniflorin ameliorates ANIT-induced cholestasis by activating Nrf2 through an PI3K/Akt-dependent pathway in rats. Phytother Res 29: 1768-1775, 2015.

31. Ma X, Zhao YL, Zhu Y, Chen Z, Wang JB, Li RY, Chen C, Wei SZ, Li JY, Liu B, et al: Paeonia lactiflora Pall. Protects against ANIT-induced cholestasis by activating Nrf2 via PI3K/Akt signaling pathway. Drug Des Devel Ther 9: 5061-5074, 2015. 
32. Sykiotis GP and Bohmann D: Stress-activated cap'n'collar transcription factors in aging and human disease. Sci Signal 3: re3, 2010.

33. Livak KJ and Schmittgen TD: Analysis of relative gene expression data using real-time quantitative PCR and the 2(-Delta Delta C(T)) method. Methods 25: 402-408, 2001.

34. Kossor DC, Meunier PC, Handler JA, Sozio RS and Goldstein RS Temporal relationship of changes in hepatobiliary function and morphology in rats following alpha-naphthylisothiocyanate (ANIT) administration. Toxicol Appl Pharmacol 119: 108-114, 1993.

35. Enomoto A, Itoh K, Nagayoshi E, Haruta J, Kimura T, O'Connor T, Harada T and Yamamoto M: High sensitivity of Nrf2 knockout mice to acetaminophen hepatotoxicity associated with decreased expression of ARE-regulated drug metabolizing enzymes and antioxidant genes. Toxicol Sci 59: 169-177, 2001.

36. Jowsey IR, Jiang Q, Itoh K, Yamamoto $M$ and Hayes JD: Expression of the aflatoxin B1-8,9-epoxide-metabolizing murine glutathione $\mathrm{S}$-transferase A3 subunit is regulated by the Nrf2 transcription factor through an antioxidant response element. Mol Pharmacol 64: 1018-1028, 2003.
37. Okawa H, Motohashi H, Kobayashi A, Aburatani H, Kensler TW and Yamamoto M: Hepatocyte-specific deletion of the keap1 gene activates $\mathrm{Nrf} 2$ and confers potent resistance against acute drug toxicity. Biochem Biophys Res Commun 339: 79-88, 2006.

38. Umemura T, Kuroiwa Y, Kitamura Y, Ishii Y, Kanki K, Kodama Y, Itoh K, Yamamoto M, Nishikawa A and Hirose M: A crucial role of Nrf2 in in vivo defense against oxidative damage by an environmental pollutant, pentachlorophenol. Toxicol Sci 90: 111-119, 2006.

39. Anwer MS: Role of protein kinase $\mathrm{C}$ isoforms in bile formation and cholestasis. Hepatology 60: 1090-1097, 2014.

40. Arisawa S, Ishida K, Kameyama N,Ueyama J,Hattori A, Tatsumi Y, Hayashi H, Yano M, Hayashi K, Katano Y, et al: Ursodeoxycholic acid induces glutathione synthesis through activation of PI3K/Akt pathway in HepG2 cells. Biochem Pharmacol 77: 858-866, 2009.

41. Lu SC: Regulation of glutathione synthesis. Mol Aspects Med 30: 42-59, 2009.

This work is licensed under a Creative Commons Attribution-NonCommercial-NoDerivatives 4.0 International (CC BY-NC-ND 4.0) License. 